\title{
Comparison of ozone profiles from DIAL, MLS, and chemical transport model simulations over Río Gallegos, Argentina, during the spring Antarctic vortex breakup, 2009
}

Takafumi Sugita et al.

Correspondence to: Takafumi Sugita (tsugita@nies.go.jp)

The copyright of individual parts of the supplement might differ from the CC BY 4.0 License. 


\section{Supplement to the paper}

In this Supplement, we examine the large difference between DIAL and MLS at 83-100 hPa. A case study is performed for October 3, 2009, when the largest difference was observed at $83 \mathrm{hPa}$ (see Figure S1). The matching MLS pairs were extracted from one day before the DIAL measurement. The locations are plotted as pluses in Figure S2. The blue and red arrows in this

5 figure indicate nighttime ( $\sim 530$ UTC) and daytime ( $\sim 1900$ UTC) paths of the MLS measurement, respectively. The numbers for each MLS location show the $\mathrm{O}_{3}$ mixing ratios of each measurement. The $\mathrm{O}_{3}$ value of the DIAL measurement was 0.4 ppmv with an sPV value of $-1.6 \times 10^{-4} \mathrm{~s}^{-1}$. The MLS line-of-sight is in the forward direction of the Aura spacecraft flight track, resulting in a nearly north-south direction (see Figure S2). The cross-track resolution is $6 \mathrm{~km}$ wide in the MLS $240 \mathrm{GHz}$ field of view. The duration of DIAL measurements on this day was approximately from 0230 UTC to 0630 UTC. Therefore, we computed the backward air mass trajectories (Tomikawa and Sato, 2005) (www.firp-nitram.nipr.ac.jp/en/) at OAPA from 0430 UTC (the middle time) and 0630 UTC (the end time) to 0230 UTC (the start time), which are shown in Figure S2 as dotted and solid lines. The trajectories were calculated using the MERRA data on the isobaric surface at $83 \mathrm{hPa}$ (see Table S1). This suggests that the DIAL-measured air mass was an average of an almost east-west direction, $\sim 800$ km horizontally.

Next, we evaluated the MLS measurements at the region that the DIAL-measured air mass originated. There were several 15 MLS measurements on October 3 at $83 \mathrm{hPa}$, as shown by crosses in Figure S2, although no measurements were found within $500 \mathrm{~km}$ of the OAPA site. The $\mathrm{O}_{3}$ values were from $0.5 \mathrm{ppmv}$ to $1.2 \mathrm{ppmv}$, which are smaller than those on October 2. Thus, the large $\mathrm{O}_{3}$ differences between DIAL and MLS observed in Figure $\mathrm{S} 1$ could be partly explained by techniques measuring different air masses. Such a difference in $\mathrm{O}_{3}$ field is also reproduced in the MIROC-CTM simulation, as shown in Figures S3 (October 2) and $\mathrm{S} 4$ (October 3). From the simulations, it is clear that there are larger $\mathrm{O}_{3}$ values over the OAPA site on October

202 than on October 3. As shown in Figure S2, the sampling volume of the model is largest, i.e., $2.8^{\circ} \times 2.8^{\circ} \times 1 \mathrm{~km}$, in the vertical at this pressure level based on the daily average. Therefore, the fine scale structure of the $\mathrm{O}_{3}$ field can not be reproduced in this model. 


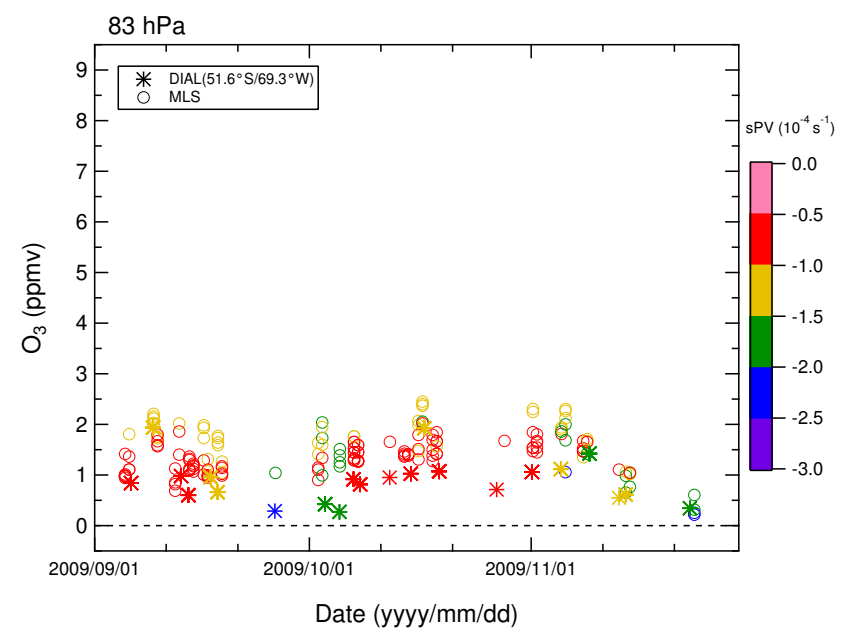

(a)

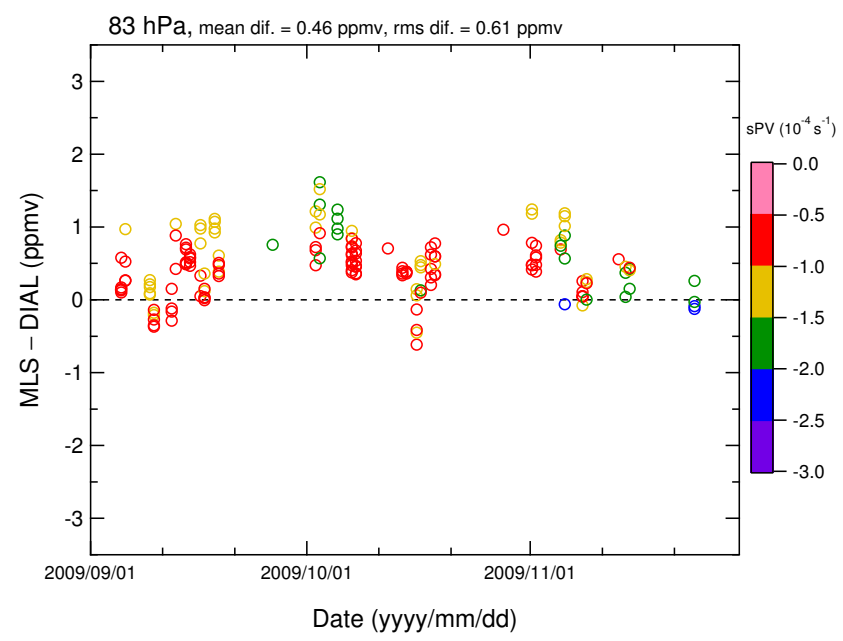

(b)

Figure S1. Time series at $83 \mathrm{hPa}$ of $\mathrm{O}_{3}$ mixing ratios measured using DIAL and MLS (a) and differences between the two (b) from September to November 2009 over the OAPA site. Data are color-coded based on SPV values. 


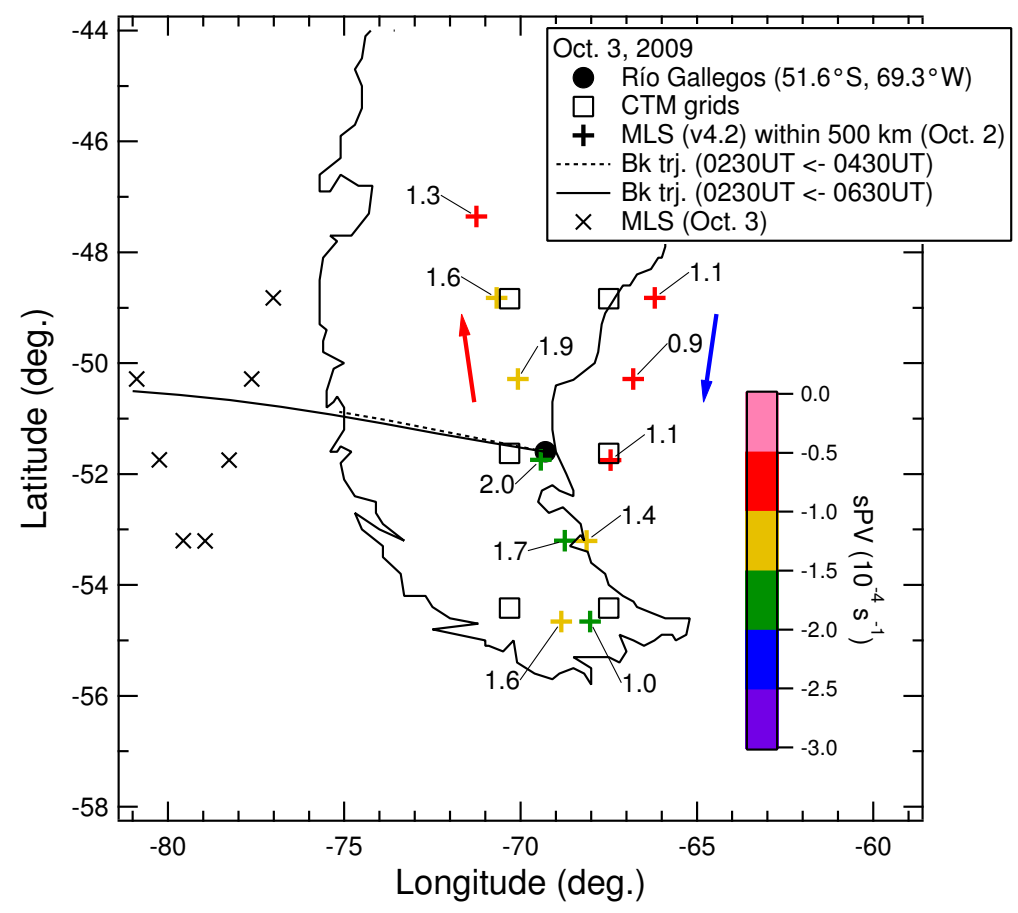

Figure S2. Locations of DIAL and MLS measurements on October 3, 2009. MLS data are color-coded based on sPV values. Numbers are $\mathrm{O}_{3}$ mixing ratios in ppmv. The six MIROC-CTM grids are also shown. The air mass trajectories from OAPA are shown as dotted and solid lines. See text for detail. 


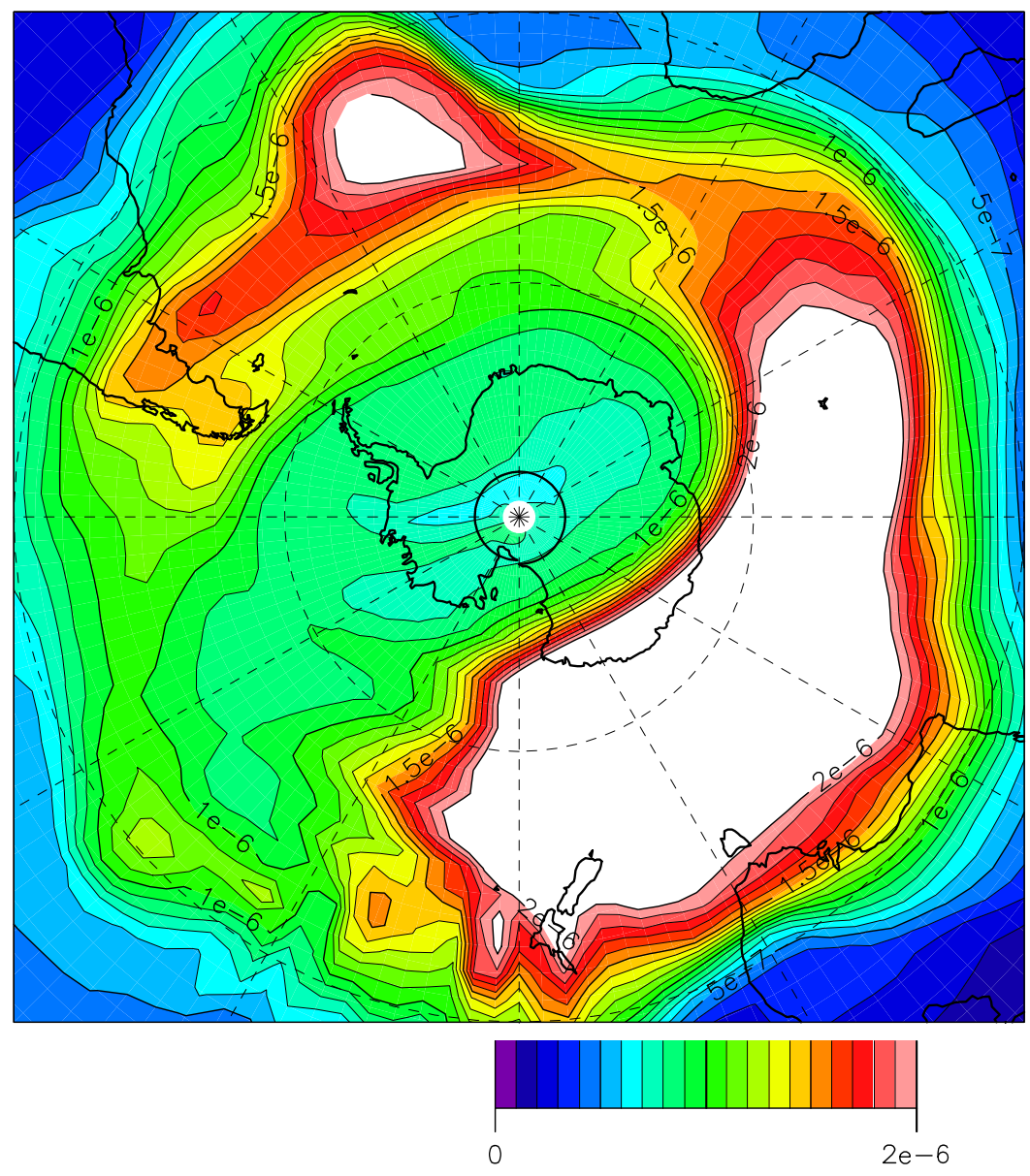

Figure S3. $\mathrm{O}_{3}$ mixing ratios at $80 \mathrm{hPa}$ computed using MIROC-CTM for October 2, 2009. The mixing ratio ranges from 0 to 2 ppmv. 

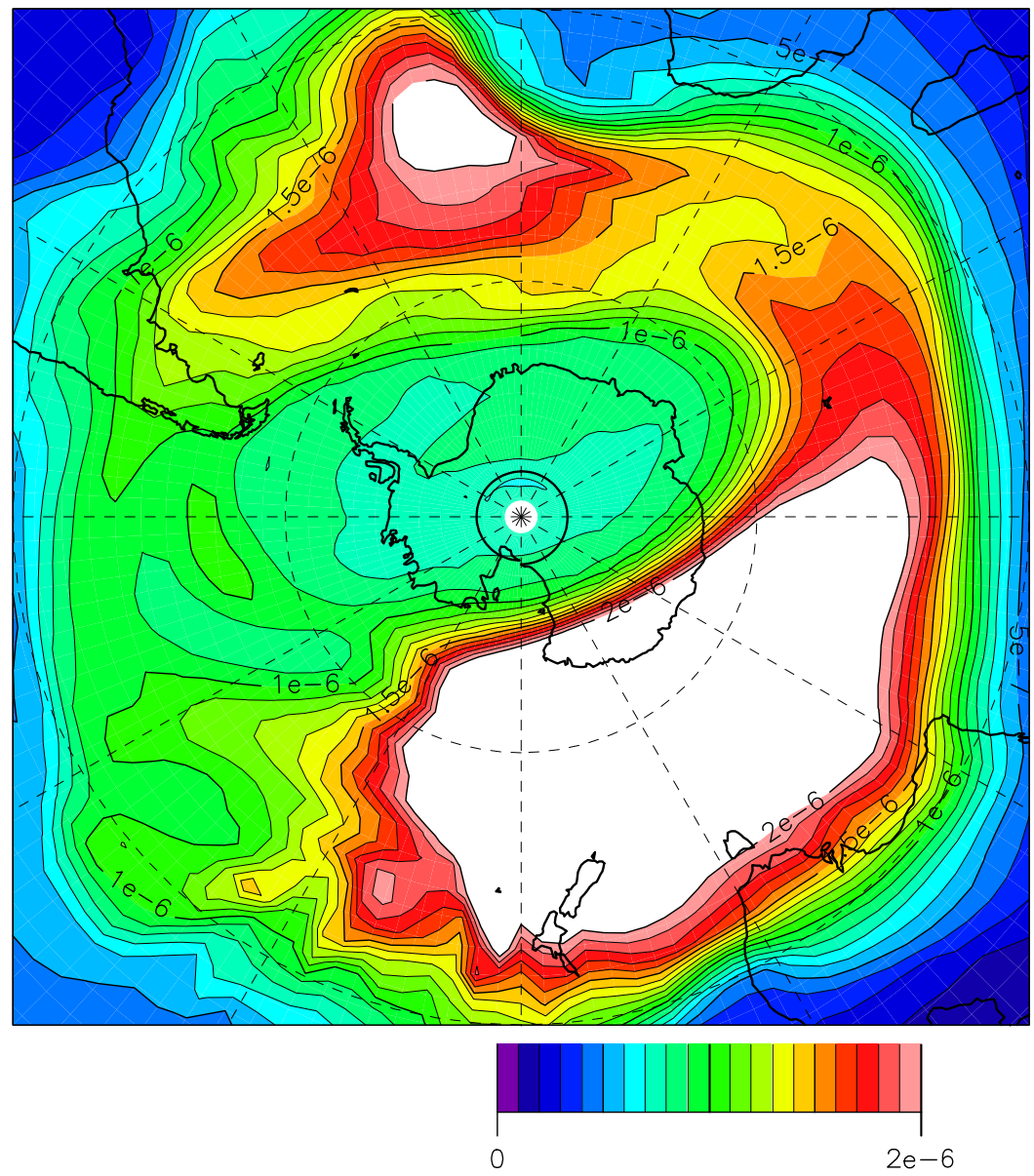

Figure S4. $\mathrm{O}_{3}$ mixing ratios at $80 \mathrm{hPa}$ computed using MIROC-CTM for October 3, 2009. The mixing ratio ranges from 0 to 2 ppmv. 
Table S1. Distances computed from air-parcel trajectory analysis from the OAPA site at the $83 \mathrm{hPa}$ level.

\begin{tabular}{ccc}
\hline $\begin{array}{c}\text { mid time of obs. } \\
\text { (yyyy/mm/dd hh:mm) }\end{array}$ & $\begin{array}{c}\text { duration } \\
\text { (hh:mm })\end{array}$ & $\begin{array}{c}\text { distance } \\
(\mathrm{km})\end{array}$ \\
\hline 2009/09/06 02:07 & $4: 20$ & 633 \\
2009/09/09 04:19 & $2: 31$ & 361 \\
2009/09/13 01:36 & $5: 23$ & 959 \\
2009/09/14 02:18 & $5: 39$ & 809 \\
2009/09/17 04:38 & $3: 25$ & 671 \\
2009/09/18 03:55 & $3: 24$ & 618 \\
2009/09/26 03:58 & $5: 45$ & 643 \\
2009/10/03 04:26 & $4: 05$ & 831 \\
2009/10/05 05:00 & $4: 29$ & 559 \\
2009/10/07 03:40 & $3: 56$ & 455 \\
2009/10/08 02:09 & $4: 58$ & 539 \\
2009/10/12 05:36 & $2: 24$ & 235 \\
2009/10/15 04:02 & $3: 21$ & 226 \\
2009/10/17 01:53 & $4: 06$ & 622 \\
2009/10/19 03:28 & $2: 54$ & 309 \\
2009/10/27 03:48 & $2: 34$ & 250 \\
2009/11/01 02:17 & $3: 06$ & 347 \\
2009/11/05 03:27 & $2: 35$ & 283 \\
2009/11/09 03:25 & $2: 54$ & 419 \\
2009/11/13 06:31 & $2: 58$ & 508 \\
2009/11/14 04:47 & $4: 41$ & 739 \\
2009/11/23 03:47 & $5: 11$ & 779 \\
2009/11/24 04:51 & $4: 12$ & 399 \\
\hline
\end{tabular}

